\title{
Nano-biomaterials in Antimicrobial Therapy
}

\author{
Pratima Parashar Pandey \\ Additional information is available at the end of the chapter \\ http://dx.doi.org/10.5772/61959
}

\begin{abstract}
Silver nanoparticles (AgNps) have attracted much interest in biomedical engineering, since they have excellent antimicrobial properties. Silver nanopolymer composites have applications in biochemical sensors, antimicrobial activity and drug delivery system. Silver nanoparticles are more effective than ionic homologues $(\mathrm{Ag}+)$ for their antimicrobial activity. Antimicrobial properties of silver nanoparticles are used by their incorporation into medical devices, tissues and other health-related products for skin pathologies to reduce the risk of contamination and to promote higher preventive infection control. Novel hybrid material thin films based on various polymeric systems with embedded silver nanoparticles were synthesized using various methods. The electrical, optical and plasmonic responses of $\mathrm{AgNps}$ onto thin layers of polymer composites show encapsulation of nanoparticles. The antibacterial activity of AgNps/polymer composites against various common bacteria is discussed in this chapter. The antibacterial activity of the synthesized hybrid materials was tested against various bacteria, commonly found in hospital environment. Silver nanostructures have especially been of interest because of contrast agents for biomedical image. Shunts used for hydrocephalous silicon elastomer grafted with hydrogel, polyvinylpyrrolidone (PVP) soaked in various antibiotics proved to be active for longer time.
\end{abstract}

Keywords: Polyvinylpyrrolidone, Silver nanoparticle, Antibacterial, Optical properties, Electrical properties, SEM

\section{Introduction}

Synthesis of nanoparticles is an interesting field in solid-state chemistry. Recently, much interest has been shown for ultrafine metal particles because they have unique properties that are different from bulk metals in optical property, catalytic activity and magnetic property. In particular, many studies have been devoted to silver, gold and copper colloids. Usually nanoparticles are spherical in shape. Nanoscale metal flakes are of interest in electronics, mechanics, electromagnetic, communication and stealth technology [1]. 
The silver antimicrobial effectiveness has been known for a long time. Over the past few years, the silver or silver salts have become important components to control the microbial proliferation. They are being currently incorporated in a wide variety of materials used in our daily lives, ranging from the textile and hospital areas to materials used in personal hygiene, such as deodorants and toothbrushes. A recent application is based on matrixes formed by collagen and bayberry tannin for the immobilization of silver nanoparticles. Various chemical forms of silver have been known for a long time as an effective antimicrobial agent with high activity against bacteria, viruses and fungi. Silver nanoparticles are most extensively studied in the recent years because of their possible usage as a strong bactericidal material with applications in the field of biomedical science. Well-developed large surface area to volume ratio of these particles provides better contact with the microorganisms and strong cytotoxicity to bacterial cells due to enough interaction with the functional groups on the bacterial cell surface [2].

Various approaches to control the size and shape of silver nanoparticles have provided many applications as antibacterial agents like medical devices, coatings for washing machines, food containers and portable water filters. In many of these applications, AgNps have been either embedded or attached on various inorganic and organic supports, such as filter materials made of zeolite, silica or fibre glass, natural macroporous materials, paper, carbon materials and various types of polymers for better contact and control for release of silver ions [3].

Various polymers such as polyglycidyl methacrylate-co-ethylene glycol dimethacrylate (polyGMA-co-EG-DMA) resins, polyvinyl alcohol (PVA) and polyvinylpyrrolidone (PVP) are used for encapsulation of silver nanoparticles.

\section{Body}

Santos et al. have shown the excellent antimicrobial effectiveness of AgNps stabilized by Pluronic ${ }^{\mathrm{TM}} \mathrm{F} 68$ associated with other polymers such as PVA and polyvinylpyrrolidone. AgNps stabilized with PVP or PVA and co-stabilized with Pluronic ${ }^{\mathrm{TM}}$ F68 are effective against Escherichia coli and Pseudomonas aeruginosa microorganisms. The wide range of minimum inhibitory concentration (MIC) from $0.78 \%$ to $25 \%$ is achieved against bacteria [4].

Wang et al. have used polyvinylpyrrolidone, which is a good stabilizer for the silver nanoparticle due to its amine functional group. PVP was used as a dispersant to accelerate the reaction between silver ions and glucose and stabilized with $\mathrm{H}^{+}$. The silver particles with the diameter $<50 \mathrm{~nm}$ coordinate with the nitrogen in PVP, by forming a protection layer. The large silver particles, with the diameter of 500-1000 nm, coordinate with both nitrogen and oxygen in PVP molecules [5].

The control of shape and size of gold particles by introducing a small amount of salt into a $\mathrm{N}, \mathrm{N}$-dimethylformamide (DMF) solution containing poly(vinylpyrrolidone) or by changing the temperature is reported in literature. The shape of gold nanoparticles has been tuned in various forms like cubes, octahedrons and hexagonal nanosheets [6]. 
Jiang et al. have explored a new method to prepare colloidal five-twinned Au nanoparticles by solvothermal wet chemical method. In this process, hydrogen tetrachloroauric acid $\left(\mathrm{HAuCl}_{2} 3 \mathrm{H}_{2} \mathrm{O}\right)$ was reduced by ethylene glycol (EG) to produce the desired shape of $\mathrm{Au}$ nanocrystals in the presence of poly(vinylpyrrolidone) molecules at $200^{\circ} \mathrm{C}$ under autogenous pressure. They also found large, five-fold twinned Au particles with perfect decahedral shape [7].

Wang et al. have shown that Ag/PVP nanocomposite fibres can be electrospun from a PVP and Ag nanoparticles ethanol solution. The pyridyl group in PVP structure have strong affinity with metal particles by undergoing hydrogen bond [8].Therefore, many metals and semiconductor nanoparticles are prepared in the PVP solution.

Washio et al. have used the idea of kinetically controlled synthesis of metal nanocrystals by using hydroxyl end group of PVP. The kinetic control is a simple and versatile route for synthesis of metal nanocrystals with well-defined shapes. They have synthesized Ag nanoplates by heating an aqueous solution of silver salt $\left(\mathrm{AgNO}_{3}\right)$ and PVP in a capped vial. The hydroxyl end groups of PVP reduced $\mathrm{AgNO}_{3}$ at a sufficiently slow rate so that the growth of Ag nanocrystals can be kinetically controlled, leading to the formation of triangular plates. This kinetically controlled synthesis gives Ag nanoplates of controllable edge lengths by varying the reaction time [9].

Polyethylene glycol (PEG) has a similar structure like polyethylene oxide (PEO). It is inexpensive, water-soluble and biocompatible. Therefore, these are used in the biomedical field for a long time. Tae et al. used these two substances (prodrug/silver) to provide a synergistic effect in both the biomedical and nanotechnology fields. Monocarboxylic ions and dicarboxylic acids are known to be very effective to stabilize silver surfaces for a long time. Vancomycin and sulfadiazine have long been known as highly active antibacterial drugs. Hence, PEO-vancomycin or PEO-sulpha drug conjugates as precursors of prodrugs, which must be effective to stabilize silver surface. Their active functional groups at the polymeric chain ends play an important role to stabilize metal surfaces by ionic-dipole or ionic-ionic interaction [10].

The polymer films of polystyrene (PS) and polymethyl methacrylate (PMMA) are technologically important for metal nanocomposites with controlled properties. Although these blend films usually result in phase separation, they are different from the bulk. This can be manipulated to produce different roughness surfaces providing different nucleation conditions for thin discontinuous metallic films. Ag nanoparticles are produced in polymer blends (PMMA/ PS) on silica substrate by e-beam evaporation of metal in vacuum [11].

Bryaskova et al. have reported that hybrid materials showed a strong bactericidal effect against E. coli, Staphylococcus aureus and P. aeruginosa and therefore have potential applications in biotechnology and biomedical science [12].

Polymeric materials such as poly(vinyl pyrrolidone) carrying amine functional group are reported to be a good stabilizer for the silver nanoparticle. The PVP is used as a stabilizer for synthesis of silver nanoparticles using two different strategies based on thermal or chemical reduction of silver ions to silver nanoparticles. The synthesized AgNps/PVP were tested against etalon strains of three different groups of bacteria: S. aureus (gram-positive bacteria), 
E. coli (gram-negative bacteria), P. aeruginosa (nonfermenting gram-negative bacteria), as well as against spores of Bacillus subtilis (B. subtilis) for antibacterial activity. Also, AgNps were tested in the presence of fungicidal activity against different yeasts and mould such as Candida albicans, Candida krusei, Candida tropicalis, Candida glabrata and Aspergillus brasiliensis. Hence, these hybrid materials show potential applications in biotechnology and biomedical science.

The chemical reduction of silver ions into stable AgNps in different polymer stabilizers is the most common method. Among them, polyvinylpyrrolidone has excellent chemical and physical properties. Therefore, it is a good material for coating or as an additive to different materials. Besides being a stabilizing agent, it also has an impact on the control of the reduction rate of the silver ions and the aggregation process of silver particles (Fig. 1). PVP is also used in other techniques for synthesis of AgNps as $\gamma$-irradiation synthesis and laser ablation [2].

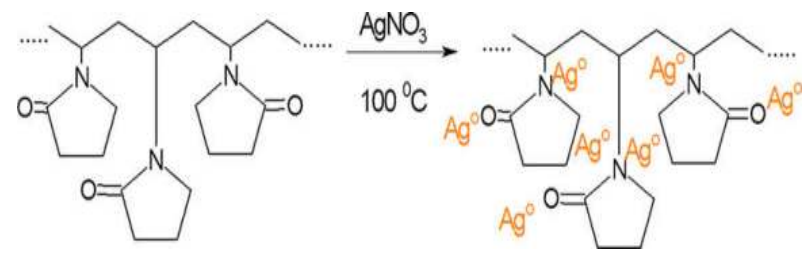

Figure 1. Chemical reduction of AgNps in PVP.

Irwin et al. have evaluated the antimicrobial properties of Ag-based nanoparticles using two solid-phase bioassays and found complete inhibition of the growth of bacteria like Staphylococcus aureus, Salmonella typhimurium or Escherichia coli on solid surfaces. Even after one week at $37^{\circ} \mathrm{C}$ on solid media, no growth was observed. At lower Np concentrations, visible colonies were observed, but they eventually ceased growing [13].

Dispersing silver nanoparticles homogeneously into a polymer matrix by ex situ methods is difficult because of the easy agglomeration of nanoparticles. Therefore, convenient and effective ways of preparing Ag nanoparticles in polymer materials are carried out by the author. Vacuum-deposited, thin discontinuous silver films on the composite of PVP and poly4vinylpyridine (P4VP) is an in situ and eco-friendly method [14].

Vacuum-deposited, thin discontinuous silver films on Eudragit RL 100 (Eu) were also produced. Eudragit RL100 is insoluble in water at physiological pH values and capable of swelling, so they are good for the dispersion of active compounds. This polymer is being used for the enteric coating of tablets and for preparing controlled-release formulations. Hence, deposition of silver nanocluster on Eu would be useful for drug delivery system [15].

Various polymer systems and methods of dispersion of silver nanoparticles in them are discussed in this chapter. A comparison is drawn among them from the point of view of applications. 


\section{Experimental}

Ivana et al. have prepared composites consisting of metallic silver nanoparticles and aminated poly(GMA-co-EGDMA) copolymer (G-NH2) by the reduction of silver ions with amino groups attached to copolymer at an elevated temperature. The content of silver in the Ag/G-NH2 composites was determined using inductively coupled plasma atomic emission measurements (ICP-AES Spectroflame 17 instrument). Bearing in mind that ICP-AES technique is not selective towards chemical state of silver, the measured value besides metallic silver includes traces of ionic silver in the composite. Further, ultraviolet--visible (UV-Vis) reflection spectra, Fourier transform infrared spectroscopy (FTIR), transmission electron microscopy (TEM) and X-ray diffraction (XRD) were used for characterization of AgNps. The antimicrobial activity of Ag/G-NH2 composites was evaluated against gram-negative bacteria E. coli (ATCC 25922), gram-positive bacteria S. aureus (ATCC 25923) and yeast C. albicans (ATCC 24433) using the standard test method (ASTM E 2149-01). The percentages of microbial reduction $(R, \%)$ after $1 \mathrm{~h}$ of contact of $\mathrm{Ag} / \mathrm{G}-\mathrm{NH} 2$ composite, in concentration range from 50 to $1 \mathrm{mg} / \mathrm{ml}$, with microbial cells were calculated using the following equation:

$$
R=\left(C_{0}-C\right) / C \times 100
$$

where $\mathrm{C}_{0}$ (CFU, colony forming units) is the number of microbial colonies in the control sample (G-NH2 copolymer without AgNps) and C (CFU) is the number of microbial colonies in the Ag/G-NH2 composites [3].

Santos et al. have used the Turkevich method for metals nanoparticle synthesis with good antimicrobial properties. This method only involves benign substances, so it is suitable for applications in the human body. This method was applied with further modifications to allow incorporation of the polymeric stabilizers and the Pluronic ${ }^{\mathrm{TM}}$ Co-stabilizer.

Wang et al. have used silver nitrate solution by dissolving in PVP, glucose and sodium hydroxide. Characterizations of the particles were achieved by TEM, infrared spectrum (IR), FTIR and extinction spectra by UV-2102 UV-Vis Spectrophotometer. All spectra were obtained from the particles immersed in water [5].

Washio et al. have applied reduction method by silver nitrate with PVP dissolved in water [9].

Tae et al. have used the PEO-sulphadiazine and the PEO-vancomycin to prepare nanosized silver particles in methanol using PEO-vacomycin prodrug as a stabilizing agent; silver nanoparticles were also obtained in aqueous media [10].

Ying et al. synthesized gold nanoparticles in DMF as both the solvent and reductant, with $\mathrm{HAuCl}_{4}$ in the presence of PVP acting as the capping agent, and these had been effectively applied for the preparation of silver nanoparticles [6].

Peng et al. have prepared colloidal, five-twinned Au nanoparticles by a solvothermal wet chemical method. Ethylene glycol (EG) is used to reduce hydrogen tetrachloroauric acid 
$\left(\mathrm{HAuCl}_{4} \cdot 3 \mathrm{H}_{2} \mathrm{O}\right)$ to produce the five-twinned Au nanocrystals. The shape of a decahedron of $\mathrm{Au}$ crystal is produced in the presence of polyvinyl pyrrolidone at $200^{\circ} \mathrm{C}$ under the condition of autogenous pressure. TEM and field emission scanning electron microscopy (FE-SEM) have been used to observe the surface morphology of the produced crystals [7].

Wang et al. have prepared the silver nanoparticles by a known quantity of silver nitrate added to ethanol containing a predetermined quantity of PVP. The molar ratio of silver nitrate to a repeating unit of PVP was 1:10. Ethanol is widely used as a solvent for the electro-spinning of polymers. Thus, Ag nanoparticles in ethanol solution were prepared by reducing Ag+ ions in a PVP solution. It has been reported that ethanol alone could reduce $\mathrm{Ag}+$ ions into $\mathrm{Ag}^{0}$ under refluxing conditions without any external reducing agents [8].

Bryaskova et al. have prepared thin films based on PVA/tetraethyl orthosilicate (TEOS) embedded with silver nanoparticles using sol-gel method. Two different strategies were adopted for the synthesis of silver nanoparticles in PVA/TEOS matrix: one on reduction of the silver ions by thermal annealing and the other by synthesis of silver nanoparticles using PVA as a reducing agent [12].

Nanosilver polymer composite was prepared by vacuum evaporation and characterised by electrical and optical properties for PVP/AgNp and Eu/AgNp [14, 15].

\section{Result and Analysis}

Various polymeric systems and methods are found in the literature for encapsulation of nanoparticle in polymer matrix. Also, the size of the nanoparticle is controlled by modifying method as per their applications.

Polymers are widely used in the chemical synthesis of colloidal nanocrystals, and their roles are generally documented as steric stabilizers or capping agent [16 - 23]. Polyvinylpyrrolidone is an excellent dispersant, biocompatible polymer. In particular, PVP has received special attention because of its high chemical stability, no toxicity and excellent solubility in many polar solvents. The role of PVP in silver capping has received considerable attention due to the chemical structure of PVP that contains $\mathrm{N}$ atom that can protect the silver particles from growing and agglomerating. With the introduction of PVP into the process, silver ions coordinate with $\mathrm{N}$ or $\mathrm{O}$ in PVP, and a covered layer would generate on the surface of the particles. The layer inhibited the growth and agglomeration of the particles [5].

For the synthesis of silver nanoparticles, organic substances, such as ethanol, DMF and formaldehyde, can be used to reduce silver. DMF is a moderate reducing agent. Silver cation was reduced and the formed carbonic acid was decomposed easily:

$$
\mathrm{HCONMe}_{2}+2 \mathrm{Ag}^{+}+\mathrm{H}_{2} \mathrm{O} \rightarrow 2 \mathrm{Ag}^{0}+\mathrm{Me}_{2} \mathrm{NCOOH}+2 \mathrm{H}^{+}
$$




$$
\mathrm{Me}_{2} \mathrm{NCOOH} \rightarrow \mathrm{CO}_{2}+\mathrm{Me}_{2} \mathrm{NH}
$$

The reaction proceeds at a meaningful rate even at room temperature and in the dark. Moreover, the reaction can be conducted without taking any special care with regard to the presence of oxygen. Reduction of silver nitrate in DMF was readily observed by the appearance of a yellow colour, which deepened to greenish-black with time. In the UV-Vis spectrum (Fig. 2), a characteristic peak for $\mathrm{Ag}^{0}$ at $415 \mathrm{~nm}$ appeared with the reduction of $\mathrm{Ag}^{+}$by DMF.

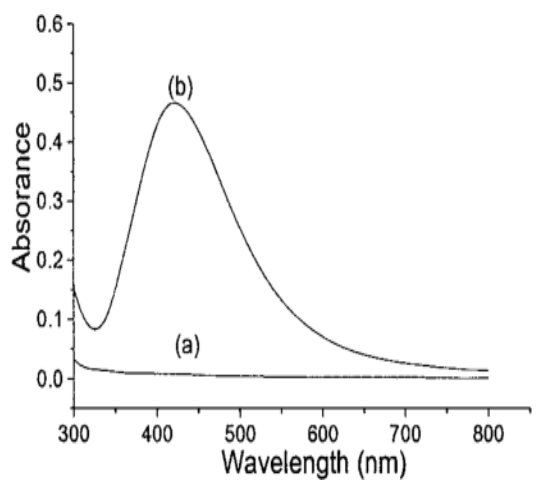

Figure 2. UV-Vis spectra for (a) $\mathrm{AgNO}_{3}^{-}$.

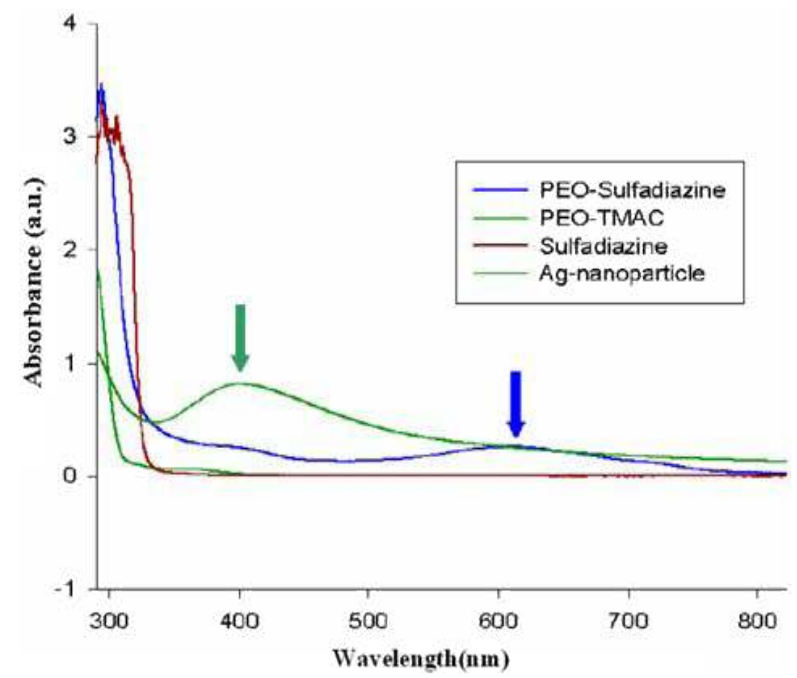

Figure 3. The electric spectral change of DMF system and (b) after $12 \mathrm{~h}$ at $60^{\circ} \mathrm{C}$. PEO- stabilized $\mathrm{Ag}$ nanoparticles and the corresponding PEO-based polymers in methanol. 
PVP, the molar ratio of vinylpyrrolidone unit to $\mathrm{AgNO}_{3}$ (10/1), was used as a stabilizer. The $\mathrm{Ag}^{+}$ions and $\mathrm{Ag}$ particles were loosely bonded to the chain of PVP to make the system stable. It was found that the average diameter of silver particles changed with the ratio of PVP unit/ $\mathrm{Ag}^{+}[1]$.

PEO-sulfadiazine is used as a stabilizing agent, while reducing $\mathrm{AgNO}_{3}$ with $\mathrm{NaBH}_{4}$ in methanol shows interesting optical properties. These optical properties of nanosized metals are resulting from their surface plasmon resonances due to collective oscillation of conduction electrons in response to optical excitation. These properties are strongly dependent on size and shape. It has been observed that Cs symmetrical nanosized spheres have only one peak, whereas $\mathrm{OH}$ symmetrical cubic particles show three peaks. Also, three optical spectral peaks in the case of triangular silver nanoplates were observed at $\lambda_{\max }=335,470$ and $690 \mathrm{~nm}$ as shown in Figure 3. The PEO-sulphadiazine conjugate exhibits the weak absorption peaks at $\lambda_{\max }=400$ and $620 \mathrm{~nm}$. But, the broad absorption peak at $\lambda_{\max }=400 \mathrm{~nm}$ in the case of PEO-sulphadiazinestabilized Ag nanoparticles in water indicate that the nanoparticles are spherical particles exhibiting symmetrical cubic lattice structure [10].

Although PVP has been widely cited as a steric stabilizer or capping agent in the synthesis of various colloidal particles, Washio et al. have used the end groups of PVP as a reducing agent for the synthesis of metal nanoparticles. The repeating unit of PVP has been extensively investigated for its coordination capability in terms of functionality and reactivity. For commercially available PVP, their ends are terminated with the hydroxyl $(-\mathrm{OH})$ group because of the involvement of water as a polymerization medium and the presence of hydrogen peroxide. The introduction of PVP accelerated the reaction between silver ions and glucose. PVP increases rate of converting ions into silver nanoparticles. If the rate of generating metal atoms is sufficiently high, then the final product takes the thermodynamically favoured shapes. As the reduction gradually decreases, the nucleation and growth turn into kinetic control and a range of shapes are produced. Metal nanostructures with sharp corners and edges are capable of generating maximum electromagnetic-field enhancement and hence make these nanoparticles attractive as substrates for surface enhanced Raman scattering (SERS) detection or other spectroscopic techniques [9].

Chen et al. have discussed that absorption of chemical species by the surface has a great impact on the surface energies. For the small particles, the ratio of surface free energy to the total energy yield about their structure. When the gold nanoparticles are prepared in the PVP solution, absorption of PVP and solvent molecules on the reduced gold particles takes place. The presence of $\mathrm{NaOH}$ or $\mathrm{NaCl}$ in the solution influenced the surface energy of various gold crystal planes due to the competitive absorption between the ions of the added salt and the original adsorbents. This influences the growth rate of various facets during the crystal growth. The evidence of the change of the surface energy of various crystal faces comes from the very few coexisting multi-twinned particles. This can be attributed to a kinetic occurrence, leading to different growth rates for the different crystal faces. However, the change of the surface energies by the adsorption of foreign species may be the origin of this kinetic problem. The adsorption abilities of chemical species vary with the temperature, and therefore, the shape can also be controlled by the reaction temperature [6]. 
PVP molecules play a very important role in the formation and evolution process of the fivetwinned Au nanoparticles. It has been found that only large spherical Au particles were produced in the reaction solution without adding PVP. When PVP is added in the ethylene glycol solution, large triangular or hexagonal Au nanoplates as well as nanorods or nanowires were produced. Figure 4 shows small decahedral Au nanoparticles along with triangular and hexagonal shapes. Figure 5 is a typical TEM image of a decahedral Au nanoparticle. A nearly perfect pentagonal shape with each angle of about $108^{\circ}$ can be clearly seen. Their sizes could be systematically controlled by changing the ratio of $\mathrm{HAuCl}_{4} / \mathrm{PVP}$. The authors suggest that PVP can act as a kinetic controller of the growth rates along various crystalline facet directions by adsorption and desorption processes [7].

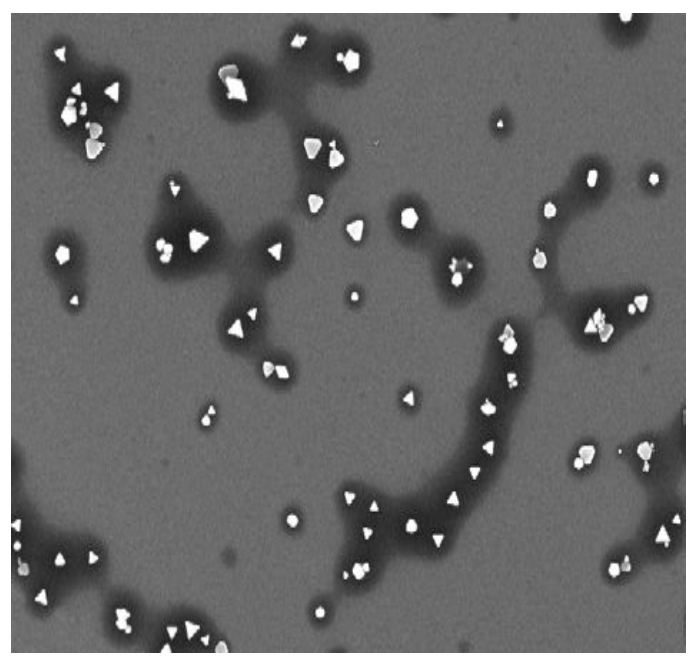

Figure 4. Decahedral Au nanoparticles.

The two major steps are involved in preparing PVP nanofibre film with homogeneously dispersed silver nanoparticles. In the first step, silver nanoparticles are prepared by reduction of $\mathrm{AgNO}_{3}$ by ethanol under refluxing conditions in PVP ethanol solution. In the second step, the solution is electro-spun directly to prepare a PVP nanofibre film. It has been reported that ethanol could reduce $\mathrm{Ag}^{+}$ions into $\mathrm{Ag}^{0}$ under refluxing conditions in the absence of any external reducing agents. The absorption spectra of the solution before and after refluxing for $5 \mathrm{~h}$ show remarkable change by reaching $410 \mathrm{~nm}$. Silver nanoparticles exhibit a high optical absorbance due to the existence of discrete energy levels which was consistent with the result of the UV absorption spectrum [8].

Two different strategies were applied for producing antibacterial hybrid material/silver nanoparticles stabilized with PVP. In comparison to other techniques for synthesis of silver nanoparticles, thermal reduction of $\mathrm{Ag}^{+}$ions to $\mathrm{Ag}^{0}$ can be performed at elevated temperature without using reducing agents. This was achieved by adding $\mathrm{AgNO}_{3}$, the precursor for silver 
ions, to the PVP solution that led to the coordination of silver ions with $-\mathrm{N}$ or $-\mathrm{O}$ atoms from PVP, followed by boiling the PVP solution at $100^{\circ} \mathrm{C}$ for $60 \mathrm{~min}$. In this case, PVP acts as a stabilizer that protects the silver nanoparticles from agglomeration and controls the rate of reduction. The optical absorption spectra clearly indicated the formation of silver nanoparticles by the appearance of strong absorption bands at $420 \mathrm{~nm}$ (Fig. 6a). Moreover, the absorption of silver nanoparticles becomes stronger and sharper with the increase in the silver concentration, which is an indication for he preparation of silver nanoparticles with narrower particles size distribution. This state is confirmed by TEM analysis that spherical silver nanoparticles with an average size of $6.4 \pm 1.1 \mathrm{~nm}$ are homogeneously distributed in polymer matrix.

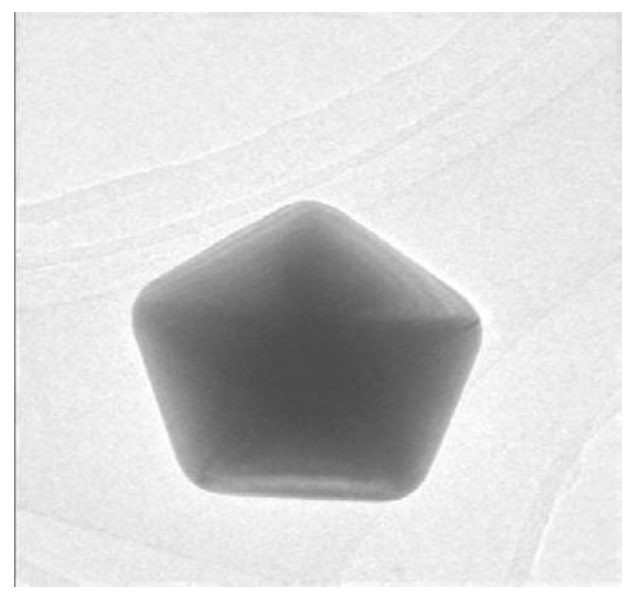

Figure 5. A typical TEM image of a decahedral Au nanoparticle.
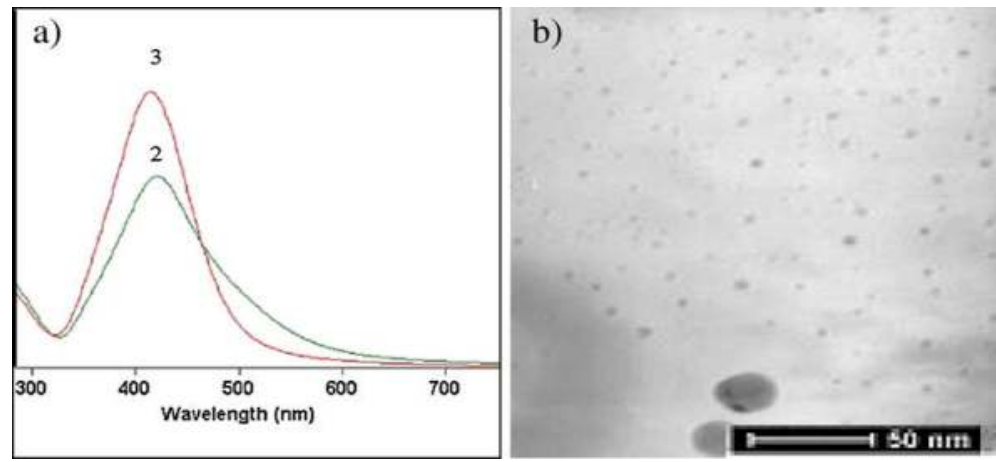

Figure 6. a) UV spectra of AgNps/ PVP obtained via thermal reduction at concentration of silver precursor: 2 for 1.96 $\mathrm{mg} / \mathrm{mL}$ and 3 for $3.92 \mathrm{mg} / \mathrm{mL}$. b) TEM of thermal-reduced silver nanoparticles stabilized by PVP at concentration of silver precursor $3.92 \mathrm{mg} / \mathrm{mL}$. 
In the second method, silver nanoparticles were prepared by adding a strong reduction agent as $\mathrm{NaBH}_{4}$ to the PVP solution in the presence of $\mathrm{AgNO}_{3}$. The optical absorption spectra of silver nanoparticles clearly show a band at $410 \mathrm{~nm}$ with peaks whose intensities increased with silver concentration (Fig. 7a). TEM analysis confirmed the formation of spherical nanoparticles with an average size of $13.8 \pm 3.8 \mathrm{~nm}$ (Fig. $7 \mathrm{~b}$ ).
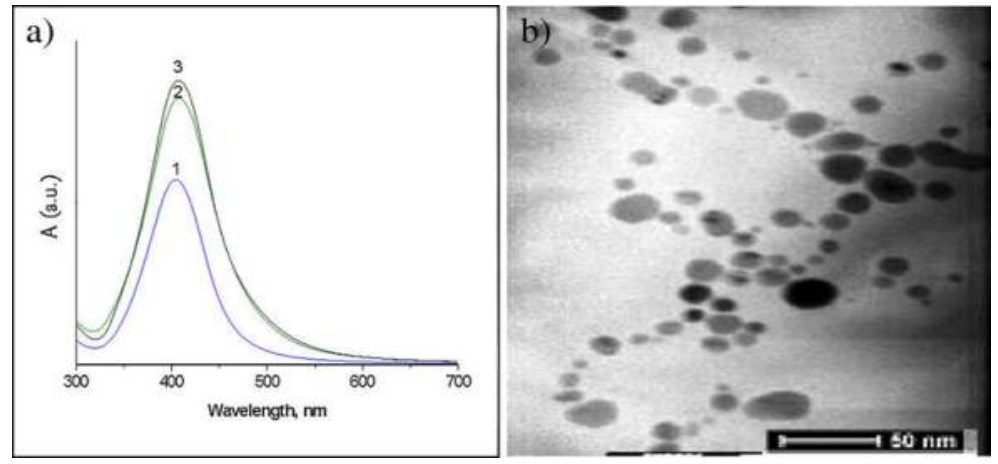

Figure 7. a) UV spectra of PVP/AgNps obtained via chemical reduction at concentration of silver precursor: 1.0 .98 $\mathrm{mg} / \mathrm{mL}, 2.1 .96 \mathrm{mg} / \mathrm{mL}$ and 3. $3.92 \mathrm{mg} / \mathrm{mL}$. b) TEM of silver nanoparticles obtained via chemical reduction of $\mathrm{AgNO}_{3}$ in the presence of $\mathrm{NaBH} 4$ at concentration of silver precursor $3.92 \mathrm{mg} / \mathrm{mL}$.

FTIR spectra of pure PVP and AgNps/PVP show characteristic peaks for $-\mathrm{CH}_{2}-\mathrm{H}-\mathrm{CH}$ groups of PVP observed in both structures. The shifting of peak for carbonyl group and $-\mathrm{C}-\mathrm{N}$ bond of PVP was associated with the formation of coordination bonds between silver atoms and oxygen or nitrogen atoms in the PVP.

Antibacterial activity of AgNps/ PVP with three different concentrations of silver precursor in the range of $0.98,1.96$ and $3.92 \mathrm{mg} / \mathrm{mL}$ was tested against $S$. aureus, E. coli, P. aeruginosa and spores of B. subtilis by disk diffusion method (DDM). It was found that all AgNps stabilized with PVP exhibit bactericidal and sporocidic activity by the appearance of an inhibition zone in the range of 7.5-8.5 mm. Higher antibacterial activity was observed against both gramnegative $E$. coli and gram-positive $S$. aureus, as well as against spores of $B$. subtilis, but lowest antibacterial activity was found for $P$. aeruginosa. To confirm the bactericidal properties, AgNps/PVP (initial concentration of silver precursor $0.98 \mathrm{mg} / \mathrm{mL}$ ) was tested using macrodilution method. By this method, the MBC corresponding to the lowest concentration without detection of bacterial growth was determined for E. coli (ATCC 25922), S. aureus (ATCC 25923) and $P$. aeruginosa (ATCC 27853). These results clearly indicated the strong bactericidal activity demonstrated by the lack of any bacterial growth even at lowest silver concentration of 0.015 $\mathrm{mg} / \mathrm{mL}$ [12].

Antibacterial PES-Ag membranes can be fabricated with PVP as dispersant in the casting solution. The effects of Ag loading and PVP (MW 360,000) on the thermal properties and contact angle revealed that membranes' contact angle decreased when Ag loading increased mainly due to hydrophilicity improvement. It was also observed that higher the concentration 
of PVP, the more hydrophilic the membrane. From the X-ray photoelectron spectroscopy (XPS) analysis, the PES-Ag membrane with $2.0 \mathrm{wt} \%$ of Ag and PVP displayed high Ag intensity, thus resulting in high activity in the antibacterial test against $E$. coli. Therefore, it is concluded that the presence of PVP in the PES-Ag membrane had significantly improved membrane performances in the antibacterial activity [19].

Irwin et al. have evaluated the antimicrobial properties of a biocompatible capping agentbased (keratin) AgNp using both solid- and solution-state media. It was found that on solid surfaces, keratin-based Nps completely inhibited the growth of Staphylococcus aureus and after several weeks at $37^{\circ} \mathrm{C}$, no further growth was observed. At lower Np concentrations, formation of colony is found but ceased growing beyond a certain small size. Growth of small colonies on fresh media without Nps proceeded normally. These results imply that further cell division is limited due to the continued presence of AgNps on the solid surface. In liquid phase, it was found that growth always occurred but the $t_{\mathrm{m}}$ varied between 7 and $>20 \mathrm{hrs}$ (assuming a constant $\left.\mathrm{C}_{\mathrm{I}}\right)$ using either the citrate- $\left([N p] \sim 3 \times 10^{-7} \mathrm{M}\right)$ or keratin-based $\left([N p] \sim 10^{-6} \mathrm{M}\right) \mathrm{Nps}$. This delay was not related to the effect that Nps had on S. aureus $k$-values. To test the possibility that the Nps were effectively changing $C_{\mathrm{I}}$ bacteria via cell death, the probabilistic calculations assumed that the perturbations in $t_{\mathrm{m}}$ were due to $\mathrm{C}_{\mathrm{I}}$ alone (i.e., with a fixed $T$ ). The observed large perturbations in $t_{\mathrm{m}}$ could only come about at concentrations where the probability for any growth occurring at all was small. This result indicates that much of the $\mathrm{Np}$-induced change in $t_{\mathrm{m}}$ was due to a greatly increased value for the true microbiological lag time ( $T$ increased from $\sim 1$ to $>15-20 \mathrm{hrs}$ ). In either solution or the solid state, a maximum perturbation was noticed only when the ratio of $[N p]: C_{I}$ (on a particle: cell basis) was about $10^{11}-10^{12}$. The differences observed between the solid and liquid growth systems relates to obvious differences in the residence time of the Nps with respect to the bacterial cell membrane [13]. A high antimicrobial effectiveness against E.coli and P. aeruginosa by AgNps was found for all stabilizing polymers, and the inhibition value was found to be increased when Pluronic ${ }^{\mathrm{TM}} \mathrm{F} 68$ was used as a co-stabilizer.. The use of AgNps co-stabilized by Pluronic ${ }^{\mathrm{TM}}$ F68 could help in the decrease of the resistance effect where the use of AgNps for burns treatment presented the onset of resistance against Entero bacteriaceas, P. aeruginosa, Salmonella and others [4].

Implantation of shunt for relief of hydrocephalus in neurosurgery is associated with infections. To prevent infection surface modified silicon elastomer polyvinylpyrrolidone grafted silicon elastomer (SEPVP) is used. PVP being a hydrogel, SEPVP soaked more in antibacterial solution results in more longevity in antibacterial activity [26].

A negative temperature coefficient of resistance (TCR) at high temperature followed by almost zero TCR at lower temperatures exhibited by the silver particulate film on polymer composite is the indication of film consisting of small particles separated by small distances. The overall resistance of film deposited on composite decreases with increase in thickness of silver films deposited on the composite. The dc resistances of the particulate film are exponentially dependent on intercluster separation or tunnelling length. Therefore, observed high resistances were attributed to very large interspacing and larger cluster sizes for PVP, which is reduced with the blending of P4VP with PVP and increasing thickness of silver film. Pyridine ring with nitrogen atom in the structure of P4VP causes the change in the morphology. Table 1 presents 
the resistance data for the silver films of various thickness deposited on polymer blend substrates held at $457 \mathrm{~K}$ with a rate of $0.4 \mathrm{~nm} / \mathrm{s}$ [14].

\begin{tabular}{lcccccc}
\hline $\begin{array}{l}\text { Polymer } \\
\text { PVP:P4VP }\end{array}$ & Thickness & $R_{\mathrm{ts}}$ & $R_{1 \text { hra }}$ & $R_{\mathrm{rt}}$ & $R_{0.5 \mathrm{~T}}$ & $R_{\text {fexp }}$ \\
\hline $0: 100$ & $52 \mathrm{~nm}$ & 1.90 & 26.87 & 27.17 & 32.8 & 72.35 \\
\hline $50: 50$ & $86.2 \mathrm{~nm}$ & 7.2 & 77.85 & 112.89 & 127.76 & $>1000$ \\
\hline $100: 0$ & $50 \mathrm{~nm}$ & 15 & 123.3 & $>1000$ & - & - \\
\hline
\end{tabular}

All resistances are measured in $\mathrm{M} \Omega / \square$.

$\mathrm{R}_{\mathrm{ts}}$ : Resistance at the time of deposit, $\mathrm{R}_{\mathrm{lhra}}$ : Resistance after $1 \mathrm{hr}$ of annealing.

$\mathrm{R}_{\mathrm{rt}}$ : Resistance at room temperature, $\mathrm{R}_{0.5 \mathrm{~T}}$ : Resistance at time of exposure at 0.5 Torr.

$R_{\text {fexp }}$ : Resistance on full exposure to atmosphere. a. Temperature: $457 \mathrm{~K}$. b. Rate:- $0.4 \mathrm{~nm} / \mathrm{sec}$.

Table 1. Various resistances of silver particulate films deposited on polymer composite

In addition, optical properties of these films show the encapsulation of silver particles. The observed shifts of the resonance position to higher wavelength (red shift) for silver particles embedded in PVP/P4VP blends were correlated with changes of particle sizes and interseparation in silver clusters. It can be clearly seen (Fig. 8) that the plasmon resonance peak shifts towards the longer wavelength side for the PVP/P4VP, 50:50 (653), as compared to pure PVP $(611 \mathrm{~nm})$ for silver film thickness of $86 \mathrm{~nm}$.

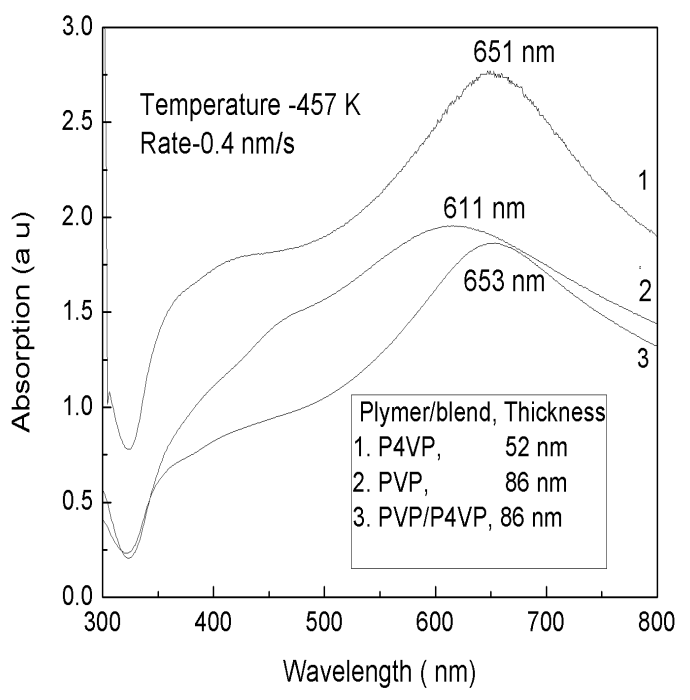

Figure 8. Optical absorption spectra for thicknesses 52 and $86 \mathrm{~nm}$ silver particulate films deposited on PVP/P4VP blends. 


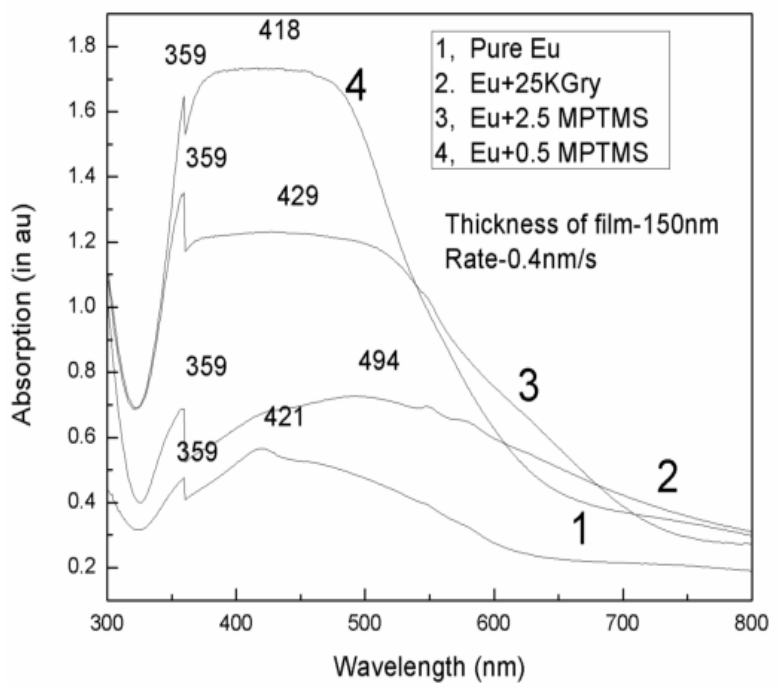

Figure 9. Optical absorption spectra for $150 \mathrm{~nm}$ silver particulate films deposited on pure Eu and its blends.

The optical absorption spectra recorded for $150 \mathrm{~nm}$ silver films deposited on polymeric blends of Eu held at $457 \mathrm{~K}$ at the deposition rate of $0.4 \mathrm{~nm} / \mathrm{s}$. For silver particles embedded in Eu blends, a higher wavelength red shift of the resonance position was found for irradiated Eu (494 nm) and Eu (+ 2.5 MPTMS) (429 nm) and negligible blue shift was found for Eu (+0.5MPTMS). These changes were correlated with changes of particle sizes and interseparation in silver clusters. It can be clearly seen (Fig. 9) that the plasmon resonance peak shifts towards the longer wavelength side as compared to pure $\mathrm{Eu}(421 \mathrm{~nm})$. Also, there is an increase in intensity of absorbing peaks that signify the decrease of particle size. Irradiation on Eu released free radicals, which interact with silver clusters resulting in decrease in size. In addition, sedating with 2.5 methacryloxy-propil-trimethoxysilane (MPTMS) resulted in decrease in size of silver clusters.

\section{Further Research}

The effect of antimicrobial activity can be studied with vacuum-evaporated silver particulate films and a comparison can be drawn.

\section{Conclusions}

Polyvinylpyrrolidone is a very useful polymer for antimicrobial activity. It is the base for most of the application. The encapsulation of silver nanoparticles is generally characterized by 
optical absorption spectra. Therefore, this is an easy and economical method for detecting silver in polymer system.

\section{Acknowledgements}

The author is thankful to DST, India, for their financial support in carrying out this research work.

\section{Author details}

Pratima Parashar Pandey*

Address all correspondence to: pratimaparashara@rediffmail.com

Applied Sciences, CET, IILM, Greater Noida, India

\section{References}

[1] Gao YU, Yuzhaoun YU: Deposition of silver nanoparticles on montmorillonite platelets by chemical plating. J. Mater. Sci.2002; 37:5083-5087.

[2] Bryaskova R, Daniela P, Stanislav N, Kantardjiev T: Synthesis and comparative study on the antimicrobial activity of hybrid materials based on silver nanoparticles (AgNps) stabilized by polyvinylpyrrolidone (PVP). J. Chem. Biol.2011; 4:185-191.

[3] Ivana D, Vukoje ES, Džunuzovic, Vesna V, Vodnik S, Dimitrijevic S, Phillip A, Jovan M, Nedeljkovic: Synthesis, characterization, and antimicrobial activity of poly (GMAco-EGDMA) polymer decorated with silver nanoparticles. J. Mater. Sci.2014; 49:68386844 .

[4] Santos CA D, Angela FJ, Adalberto P, Marcelo, Martins S: Antimicrobial effectiveness of silver nanoparticles co-stabilized by the bioactive copolymer pluronic F6. J. Nanobiotechnol.2012; 10:43-49.

[5] Hongshui W, Xueliang Q, Jianguo C, Xiaojian W, Shiyuan D: Mechanisms of PVP in the preparation of silver nanoparticles. Mater. Chem. Phys.2005; 94:449-453.

[6] Ying C, Xin G, Cha-Geng N, Zhi-Yuan J, Zhao-Xiong X, Chang- Jian L: Shape controlled growth of gold nanoparticles by a solution synthesis. Chem. Commun.2005; 4181-4183. 
[7] Peng J, Jian-Jun Z, Rui L, Zhong-Lin W, Si-Shen Xie: Pol(vinyl pyrrolidone)- capped five-fold twinned gold particles with sizes from nanometres to micrometres. Nanotechnology.2006; 17:3533-3538.

[8] Yongzhi W, Yaoxian L, Songtao Y, Guangliang Z, Dongmin A, Wang C, Qingbiao Y, Xuesi C, Xiabin J, Yen W: A convenient route to polyvinyl pyrrolidone/silver nanocomposite by electrospinning. Nanotechnology.2006; 17:3304-3307.

[9] Isao W, Yujie X, Yadong Y, Younan X: Reduction by the end groups of poly (vinyl pyrrolidone): A new and versatile route to the kinetically controlled synthesis of Ag triangular nanoplates. Adv. Mater.2006; 18:1745-1749.

[10] Tae HK, Keunsuk K, Geon HP, Jin HC, Sangmi L, Ho-Jung K, Jae YL, Jungahn K: Preparation and characterization of water-dispersible silver nanoparticles stabilized by PEO-conjugated pro-drugs. Macromol Res.2009; 17(10):770-775.

[11] Pui`soa J, Prosy`cevasb I, Guobien eb A, Tamulevi`ciusa S: Mater Sci Eng. B.2008; 149:230-236.

[12] Bryaskova R, Pencheva D, Kale GM, Lad U, Kantardjiev T: J. Colloid Interface Sci. 2010; 349(1):77-85.

[13] Irwin P, Martin J, Ly-Huong Nguyen LH, Yiping H, Gehring A, Yi Chen C: Antimicrobial activity of spherical silver nanoparticles prepared using biocompatible macromolecular capping agent: evidence for induction of a greatly prolonged bacterial lag phase. J. Nanobiotechnol.2010; 8:34-46.

[14] Parashar P: Synthesis of silver nanocomposite with poly(vinylpyrollidone) and poly(4-vinylpyridine) for antimicrobial activity. Advanced. Materials Res.2013; 772:9-14.

[15] Parashar P: Synthesis of silver nanocomposite with EUDRAGIT RL100 for the drug delivery system. Int. J. Edu. Appl. Sci. Res.2014; 3(1): 51-54.

[16] Duff DG, Baiker A, Edwards P: Langmuir.1993; 9:2301.

[17] Agt PY, Urbina RH, Elhsissen KT: J. Mater. Chem.1997; 7:293.

[18] Pastoriza-Santos I, Liz-Marzán LM: Nano Lett. 2002; 2:903.

[19] Chen J, Herrick T, Geissler M, Xia Y: J. Am. Chem. Soc.2004; 126(10):854.

[20] Xiong Y, Chen J, Wiley B, Xia Y, Aloni S, Yin Y: J. Am. Chem. Soc.2005; 127:7332.

[21] Sun Y, Xia Y: Science.2002; 298:2176.

[22] Wiley B, Herricks T, Sun Y, Xia Y: Nano Lett.2004; 4: 1733.

[23] Wiley B, Sun Y, Mayers B, Xia Y: Chem. Eur. J.2005; 11:455. 
[24] Petroski JM, Wang ZL, Green TC, El-Sayed MA: Kinetically controlled growth and shape formation mechanism of platinum nanoparticles. J. Phy. Chem. B.1998; 102:3316- 3320 .

[25] Basri H, Ismail AF, Aziz M: Assessing the effect of PVP of various molecular weights (MW) in PES-Ag membranes: Antimicrobial study using E.Coli. J. Sci Technol.2011; 3(2):59-67.

[26] Boelean JJ, Tan WF, Dankert J, Zaat SAJ: Antibacterial activity of antibiotic soaked polyvinylpyrrolidone-grafted silicon elastomer hydrocephalus shunt. J. Antimicrob. Chemother.2000; 45:221-224. 
